\title{
Desafios da adesão à dieta sem glúten
}

\author{
Challenges in adhering the gluten-free diet \\ Desafíos de adherirse a la dieta sin gluten
}

\section{Resumo}

A dieta sem glúten é utilizada recorrentemente como estratégia para o tratamento de diversas doenças. No entanto, as restrições provocadas por ela a tornam desafiadora, levando a altos percentuais de não adesão. Assim, o presente estudo tem o objetivo de discorrer sobre os diversos fatores que podem prejudicar a adesão à dieta livre de glúten. Para isso, foi realizada uma revisão bibliográfica qualitativa com os descritores dieta, sem glúten, adesão à dieta, adesão ao tratamento e desafios, nas bases de dados Scielo, Pubmed, Science.gov, Scopus e Google Acadêmico. Foram encontradas diversas origens para a não adesão à dieta sem glúten, dentre elas a falta de conhecimento do consumidor e dos manipuladores de alimentos, inadequações nos rótulos e falta de conhecimento na leitura dos mesmos, contaminação cruzada e alto custo dos alimentos sem glúten, falta de disponibilidade e acesso a esses alimentos e características sensoriais desagradáveis, além de questões de natureza individual e social. Diante disso, evidencia-se a variedade de fatores interferentes na adesão e a necessidade de atendimento individualizado como meio de monitoramento à adesão, para que se apliquem intervenções assertivas. Ademais, novos estudos devem focar em intervenções, para que se melhorem os aspectos abordados e, por conseguinte, a autonomia dessas pessoas que necessitam seguir a dieta sem glúten.

Palavras-chave: Adesão à dieta; Desafios; Sem glúten.

\begin{abstract}
The gluten-free diet is recurrently used as a strategy for the treatment of various diseases. However, the restrictions caused by it make it challenging, leading to high percentages of non-adherence. Thus, the present study aims to discuss the various factors that could impair adherence to a gluten-free diet. In this regard, a qualitative bibliographic review was conducted with the descriptors diet, gluten-free, diet adherence, treatment adherence, and challenges, in the following databases Scielo, Pubmed, Science.gov, Scopus, and Google School. Several sources were found for non-adherence to the gluten-free diet, among them: insufficient information of the consumer and food handlers, inadequacies in labels and lack of knowledge in reading them, cross-contamination, and high cost of gluten-free food, lack of availability and access to these foods and unpleasant sensory characteristics, in addition to other aspects of an individual and social nature. In this light, the variety of factors interfering in the adherence and the need for individualized care as an instrument of monitoring adherence are evident, so that assertive interventions can be applied. Moreover, further studies should focus on interventions to improve the aspects addressed and, therefore, the autonomy of these people who need to follow a gluten-free diet.
\end{abstract}

Keywords: Challenges; Dietary adherence; Gluten-free.

\section{Resumen}

La dieta libre de gluten se utiliza de manera recurrente como estrategia para el tratamiento de diversas enfermedades. Sin embargo, las restricciones que provoca lo hacen desafiante, lo que lleva a altos porcentajes de incumplimiento. Por lo tanto, el presente estudio tiene como objetivo discutir los diversos factores que pueden perjudicar la adherencia a una dieta sin gluten. Para ello, se realizó una revisión bibliográfica y cualitativa con los descriptores dieta, sin gluten, adherencia a la dieta, adherencia al tratamiento y desafíos, en las bases de datos académicas Scielo, Pubmed, Science.gov, Scopus y Google Académico. Se encontraron varias fuentes de no adherencia a la dieta sin gluten, entre ellas el desconocimiento del consumidor y manipuladores de alimentos, insuficiencias en las etiquetas y desconocimiento en la lectura de las mismas, contaminación cruzada y alto costo de los alimentos sin gluten, falta de 
disponibilidad y acceso a estos alimentos y características sensoriales desagradables, así como cuestiones de carácter individual y social. Frente a eso, se evidencia la variedad de factores que interfieren en la adherencia y la necesidad de atención individualizada como forma de supervisar la adherencia, para que se puedan aplicar intervenciones asertivas. Además, futuros estudios deberían centrarse en intervenciones para mejorar los aspectos abordados y, por tanto, la autonomía de estas personas que necesitan seguir una dieta sin gluten.

Palabras clave: Adherencia a la dieta; Desafíos; Sin Gluten.

\section{Introdução}

O glúten é uma proteína de armazenamento insolúvel presente principalmente em cereais como o trigo, centeio e cevada (Cozzolino, 2016), entretanto ele pode ser encontrado também em outros cereais e farinhas, em razão da contaminação capaz de ocorrer nos processos de colheita, transporte e armazenamento (Demirkesen et al., 2020).

Essa proteína é formada por tipos de prolaminas, a depender do cereal, e por glutenina (Khoury et al., 2018); quando misturadas com água e são submetidas a um trabalho mecânico, formam, nas farinhas que as contém, uma rede viscoelástica. No caso do trigo isso ocorre por causa da atuação plastificante da gliadina, tipo de prolamina, que, juntamente com a resistência propiciada pela glutenina, confere às massas um obstáculo à deformação decorrente da pressão ocasionada pelo $\mathrm{CO}^{2}$ quando a massa está em processo de crescimento (Borin et al., 2018; Domínguez, 2017).

A dieta sem glúten (DSG) é caracterizada pela retirada de alimentos que contém glúten, o que pode torná-la bastante restritiva, já que acaba excluindo grande parte das fontes de carboidrato. Para se ter uma ideia, atualmente o glúten é responsável por até 50\% do valor energético consumido em países desenvolvidos e em desenvolvimento (Tovoli et al., 2015). Dessa forma, a DSG acaba sendo uma mistura de alimentos naturalmente livres de glúten, tais como arroz e batata, com as versões sem glúten dos produtos alimentícios, tais como pães e massas em geral (Muhammad et al., 2017).

Essa dieta é largamente utilizada por indivíduos portadores da doença celíaca (DC), portadores de sensibilidade ao glúten, portadores de alergia ao glúten e de diversos outros distúrbios que apresentam respostas mediadas pelo glúten (Rostami et al., 2017). Entretanto, nos últimos anos tem se difundido a ideia de que a DSG pode promover também benefícios para pessoas que não possuem problemas com o glúten (Khoury et al., 2018). Em conformidade com essa visão, um estudo apontou que 37\% das pessoas sentiam que a dieta livre de glúten era mais saudável, enquanto 31\% acreditavam de fato nisso (Dunn et al., 2014). Dentre os benefícios atribuídos à DSG estão o emagrecimento, a melhora do perfil metabólico e nutricional e até mesmo a melhora do sono (Cozzolino, 2016).

Mesmo que a DSG seja uma forma de tratamento imprescindível para os indivíduos com distúrbios relacionados ao glúten, especialmente para paciente celíacos, cuja dieta é a única forma de tratamento atual, as evidências têm mostrado uma adesão insuficiente à dieta (Muhammad et al., 2019), sendo fatores como custo, conhecimento, sabor, disponibilidade de alimentos, questões sociais, entre outros, citados com frequência como fatores interferentes na dieta (MacCulloch \& Rashid, 2014). Ademais, alguns estudos chegam a encontrar de $36 \%$ a $96 \%$, o que pode variar de acordo com o método utilizado para determinar a adesão (Muhammad et al., 2017).

Dessa forma, este estudo discorre a respeito dos desafios encontrados ao se adotar uma DSG, contribuindo com a criação de estratégias eficazes para contorná-los. Além disso, fornece evidências para os profissionais de saúde acerca dos problemas enfrentados pelos indivíduos que se utilizam dessa dieta, facilitando a prática de um atendimento mais humanizado e eficaz, e subsídios valiosos para os produtores de alimentos sem glúten, de forma que estes possam aprimorar suas práticas de produção e a variedade de ofertas com valores mais acessíveis, gerando impacto no acesso do consumidor. 


\section{Metodologia}

O presente estudo trata-se de uma pesquisa bibliográfica, que é construída por meio de revisão na literatura já existente. É qualitativa, ou seja, capaz de relacionar a realidade com o objeto de estudo, entendendo a condição humana nos diferentes contextos (Bengtsson, 2016). Neste caso, o objeto deste estudo trata da não adesão à dieta sem glúten, buscando apresentar e compreender a realidade das pessoas que deveriam fazer uso dessa dieta. Por fim, esta pesquisa é descritiva, pois se caracteriza pela transcrição, seja de pessoas, situações ou acontecimentos, que serão subsídio para a formação e elucidação do ponto de vista defendido (Sant'Ana et al., 2018).

Realizada no período de junho a dezembro de 2021, essa pesquisa analisou qualitativamente referências bibliográficas dos últimos 5 anos. Lançou-se mão ainda, de forma complementar, de artigos mais antigos, visto que os mesmos se tratam de embasamento teórico para as referências analisadas aqui prioritariamente. Foram utilizadas as bases de dados Scielo, Pubmed, Science.gov, Scopus e Google Acadêmico. A pesquisa foi realizada utilizando-se do método de operadores booleanos com os termos combinados em inglês e português: dieta, sem glúten, adesão ao tratamento, adesão à dieta e desafios. Foram encontrados 194 artigos e no total foram selecionados 49.

Além disso, os artigos incluídos estão na versão integral em inglês, português ou espanhol e os excluídos tratam-se de estudos repetidos, bem como de estudos que não atendiam aos objetivos do trabalho. Foram utilizados, ainda, filtros nas bases de dados para selecionar os artigos publicados nos últimos 5 anos; em seguida, foi realizada a respectiva leitura e análise dos títulos e resumos e, na sequência, os textos selecionados passaram por uma segunda triagem, realizada por meio da leitura completa dos mesmos, a fim de identificar aqueles que se encaixavam de fato nos objetivos do trabalho.

\section{Resultados e Discussão}

\subsection{O conhecimento do consumidor sobre a dieta}

O conhecimento sobre o que envolve uma DSG, para evitar equívocos e exposição não intencional ao glúten, é essencial para melhorar a adesão à dieta livre de glúten; especialmente para aqueles que a utilizam como forma de tratamento, já que a exposição a longo prazo pode gerar danos irreversíveis à mucosa intestinal. Dessa forma, compreender não só a própria condição, mas também a importância e em que consiste a dieta, está diretamente ligado à maior adesão a ela (Paganizza et al., 2019).

Estudos têm apontado que algumas pessoas, apesar de relatarem possuir uma dieta restrita de glúten, demonstram, através de questionários, estar suscetíveis ao consumo inadvertido do mesmo, devido à falta de conhecimento em identificar alimentos sem glúten, o que pode até levá-las a restringir o consumo de alimentos de maneira desnecessária, aumentando os riscos de deficiência nutricional (Gutowski et al., 2020; Jamieson et al., 2019; Silvester et al., 2016).

Vale ressaltar que até mesmo a exposição intencional ao glúten parece estar relacionada ao baixo nível de conhecimento a respeito da DSG, podendo estar associada ainda à menor motivação em identificar os produtos que contém ou não essa proteína, também decorrente dessa falta de conhecimento (Jamieson et al., 2019).

Para verificar o conhecimento a respeito da dieta livre de glúten, um estudo realizado com 57 participantes verificou que a ingestão de produtos à base de cereais de uma forma geral não foi considerada como uma exposição óbvia ao glúten, indicando falta de conhecimento, já que a contaminação cruzada é comum nesses alimentos. Esse estudo ressalta, portanto, a necessidade de um conhecimento genuíno do conteúdo de glúten dos alimentos, tendo o rótulo um importante papel para tornar esse conhecimento mais acessível (Løvik et al., 2017).

Além disso, o monitoramento da adesão à dieta é essencial para verificar as causas de sintomas persistentes. Para isso, diversos métodos podem ser utilizados, tais como biópsia, questionários, percepção de sintomas, etc. Alguns são mais 
eficazes que outros e mais caros, sendo um importante fator de escolha que os métodos tenham alta especificidade para monitorar adequadamente essa exposição. Ademais, pode-se recorrer também ao acompanhamento nutricional e psicológico e até mesmo a ações de educação para que a barreira do conhecimento deixe de ser um fator relevante na não adesão inadvertida à DSG (Wieser et al., 2021).

\subsection{Os rótulos dos alimentos}

A leitura correta dos rótulos dos alimentos é uma prática que exige um tempo maior no momento da compra, podendo tornar essa tarefa extenuante (Oyarzún et al., 2015). Entretanto, ela é de extrema importância, especialmente para pacientes celíacos, pois é capaz de evitar a contaminação com a proteína do glúten (Madden et al., 2016). Em um estudo realizado no Canadá com 144 participantes, 76\% relataram exposição acidental ao glúten, sendo isso reflexo não só da falta de conhecimento na leitura dos rótulos, mas também na ambiguidade que muitas vezes pode estar presente neles, dificultando ainda mais a identificação eficaz dos alimentos. Além disso, esse trabalho evidencia que a prática de colocar nos rótulos, de forma explícita, se há ou não glúten, ainda que não seja capaz de erradicar a ingestão inadvertida de glúten, demonstra reduzir erros na escolha dos alimentos (Gutowski et al., 2020).

Um estudo transversal relacionou o conhecimento de leitura de rótulos com a adesão à DSG. Nele, $73 \%$ das pessoas que não sabiam ler rótulos foram classificadas como não aderentes à dieta. Se observou ainda que, das pessoas que não receberam prescrição de comidas sem glúten por um profissional, 62\% não seguiam a dieta, demonstrando que com os gargalos de conhecimento atual é ainda mais necessário que se busque orientação do profissional de nutrição (Muhammad et al., 2017).

Apesar disso, é importante salientar que o Brasil já possui leis de obrigatoriedade à identificação explícita quanto ao conteúdo de glúten, e mesmo quando não há presença dessa proteína é, por lei, necessário indicar como "sem glúten” (Lei n. 10.674, 2003).

\subsection{O conhecimento dos manipuladores de alimentos}

Trabalhar com a alimentação, especialmente com cardápios onde há restrições alimentares requer um grande cuidado e conhecimento por parte dos profissionais, a fim de evitar intercorrências na saúde dos consumidores (Case, 2005). Dessa forma, não é suficiente apenas o conhecimento do indivíduo que faz uso da DSG. Por isso, tem-se estudado e avaliado o nível de conhecimento de chefs e cozinheiros nesse quesito.

Um estudo realizado na Nova Zelândia com 90 restaurantes que afirmaram fornecer opções sem glúten, mostrou que mesmo quando os participantes afirmaram saber sobre a DC, tal fato não implicou necessariamente em maior conhecimento para determinar quais alimentos possuem ou não glúten, nem na adoção de políticas adequadas para prevenção de contaminação. No mesmo estudo também se identificou que estudantes de culinária vêm recebendo treinamento e apresentaram maior conhecimento quanto às preparações livres de glúten (Schultz et al., 2017).

Deste modo, fica evidente a necessidade de cuidado e conhecimento por parte de quem não pode consumir glúten, especialmente ao comer fora, e ainda a emergência de treinamentos para os profissionais da área.

\subsection{Sobre os produtos sem glúten}

\subsubsection{Contaminação cruzada}

A contaminação cruzada é apontada como uma das principais causas da não adesão inadvertida à dieta (Wieser et al., 2021). Um estudo chegou à conclusão de que cerca de $13 \%$ dos produtos rotulados como sem glúten e 41,5\% dos produtos oferecidos por serviços de alimentação ultrapassaram $20 \mathrm{mg} / \mathrm{Kg}$, sendo esse o limite máximo de glúten que um alimento pode 
conter para ser considerado seguro segundo o Codex Alimentarius (Codex Alimentarius Comission, 2008; Falcomer et al., 2018). Não é incomum que os alimentos certificados como sem glúten ainda apresentem um baixo teor dessa proteína, raramente ultrapassando $100 \mathrm{mg} / \mathrm{Kg}$, quantidade essa que pode acarretar graves problemas para paciente celíacos. Por isso, medicamentos contendo glúten oculto, alimentos considerados naturalmente sem glúten e produtos alimentícios de forma geral são considerados perigos em potencial para pacientes com DC, visto que há uma probabilidade razoável de que estes produtos apresentem quantidades de glúten maiores do que $100 \mathrm{mg} / \mathrm{kg}$. Este perigo pode ainda ser acentuado pelo fato de o consumo destes ser de difícil identificação como responsáveis pela não adesão (Wieser et al., 2021).

Apesar do Codex Alimentarius ter sugerido um valor tolerável, essa flexibilidade vai depender de cada indivíduo. Enquanto uns toleram cerca de 34-36 mg ao dia, outros desenvolvem anormalidades na mucosa por consumir $10 \mathrm{mg}$ de glúten ao dia e, embora não haja evidências, assume-se que a ingestão menor que 10 mg diária não acarretará em prejuízos na mucosa do indivíduo (Akobeng \& Thomas, 2008). Dessa forma, de acordo com o Codex Alimentarius, a ingestão de 500g de alimentos contendo 20 ppm (mg/Kg) seria considerada segura, pois não extrapola o limite de $10 \mathrm{mg} /$ dia (Farage et al., 2019).

Um estudo realizado no Brasil encontrou uma pequena ocorrência $(2,8 \%)$ de contaminação cruzada nas refeições oferecidas em serviços de alimentação, que estavam acima de 20 ppm (Farage et al., 2019); entretanto, no ramo da panificação, outro estudo chegou a encontrar $21,5 \%$ de contaminação, e isso se deve provavelmente ao fato de haver um maior contato com a farinha de trigo prevalecente nestes ambientes (Farage et al., 2017).

\subsubsection{Custo}

Um fator bastante considerado como interferente na adesão à dieta livre de glúten é o custo desses produtos (Singh \& Whelan, 2011). Um estudo verificou a variedade de valores a partir do tipo de produto e do tipo de mercado onde ele é vendido, considerando que este se ajusta ao nível socioeconômico da clientela que comumente atende. Verificou-se que independente das categorias os produtos sem glúten (PSG) são mais caros que seus correspondentes com glúten, sendo que alguns produtos de panificação chegaram a ser 300\% mais caros que as versões correspondentes com glúten (Oyarzún et al., 2015).

Além disso, outro estudo identificou que 50\% dos participantes não considerou o custo um fator limitante para seguir a DSG, entretanto houve correlação forte entre considerar o custo um empecilho e ter uma renda familiar média inferior (Villafuerte-Galvez et al., 2015). Dessa forma, evidencia-se a necessidade de ajuda governamental com os custos de produtos prescritos para aumentar a adesão à dieta, diminuindo as chances de complicações decorrentes do não cumprimento da mesma como tratamento, e, assim, reduzir despesas a longo prazo para o sistema de saúde (Rostami et al., 2017).

É possível que os altos preços dos PSG estejam associados ao valor dos grãos utilizados para substituir o trigo, ao alto grau de conhecimento científico necessário para o desenvolvimento desses produtos e ao valor dos aditivos utilizados nesses produtos para aproximar as suas características daquelas dos produtos feitos com trigo. Além disso, PSG costumam ter um menor tempo de prateleira, possuem menor oferta e demanda (Hanci et al., 2019) e precisam de linhas de produção separadas, para evitar a contaminação cruzada, sendo estes os fatores que também podem contribuir para os altos preços destes produtos (Demirkesen et al., 2020). Os estudos chegaram a encontrar produtos $518 \%$ mais caros que a versão convencional (Singh et al., 2011).

\subsubsection{Disponibilidade e acesso}

A baixa disponibilidade de PSG é desafiadora não só para aqueles com menor poder aquisitivo, mas também para os que vivem em cidades remotas (Khoury et al., 2018), impactando não só na adesão à dieta, mas também na qualidade de vida dessas pessoas (Hanci et al., 2019). 
A disponibilidade de alimentos sem glúten é geralmente limitada quando comparada às versões com glúten, independentemente do nível socioeconômico do mercado (Oyarzún et al., 2015; Singh et al., 2011). Entretanto, um estudo verificou que os PSG da categoria dos laticínios não apresentam diferença significativa na disponibilidade (Oyarzún et al., 2015).

Um estudo concluiu que uma média de $41 \%$ dos alimentos eram encontrados na versão sem glúten, e, dependendo do mercado, essa disponibilidade variava entre $9 \%$ e $90 \%$, sendo que os mercados mais caros ofereciam mais opções sem glúten que os baratos. Outro tipo de mercado onde também foi mais comum encontrar opções sem glúten foram os de produtos saudáveis (Singh et al., 2011). Isso evidencia a suscetibilidade de determinados grupos, como os de menor renda, que podem não aderir à dieta adequadamente, já que costumam ser mais propensos a comprar em mercados menores (Ellaway et al., 2000), que além de ter menos opções, custam mais caro (Singh et al., 2011).

Para se ter ideia do impacto que essa limitada oferta de PSG pode ocasionar no acesso, em um estudo realizado por meio de um questionário on-line, acessado no site da Associação dos Celíacos do Brasil-Santa Catarina (ACELBRA-SC), 38\% de pessoas com DC que afirmaram consumir glúten, o faziam por falta de alternativas. Além disso, 54\% das pessoas desse estudo afirmaram ter dificuldades em encontrar PSG (do Nascimento et al., 2014).

Nos últimos anos a disponibilidade dos alimentos sem glúten tem aumentado, não só em decorrência da maior popularidade dessa dieta até mesmo entre pessoas sem distúrbios relacionados ao glúten (Kim et al., 2016), mas também pela crescente tendência de compras on-line, fazendo com que, nos últimos anos, as vendas desses produtos aumentassem substancialmente (Terazono, 2017).

Outro estudo confirmou que nos últimos anos houve um aumento na disponibilidade dos alimentos sem glúten; entretanto, isso não foi significativo nos pequenos mercados, prejudicando a população desfavorável economicamente, os idosos e os deficientes físicos. Além disso, verificou-se que a possibilidade de comprar on-line não necessariamente aumentou o acesso a PSG, já que existem outras barreiras de acessibilidade a esses produtos, tais como alfabetização digital deficiente e acesso precário à internet (Hanci et al., 2019).

\subsubsection{Características Sensoriais}

Um dos principais aspectos que influenciam na decisão de compra de PSG é o sensorial (do Nascimento et al., 2014). Esses produtos costumam apresentar sabor, e textura diferentes da versão com glúten, pois costumam apresentar menor elasticidade, extensibilidade e volume, características estas atribuídas à atividade do glúten (Wieser, 2007). Apesar disso, mudanças tecnológicas já vêm sendo realizadas, fazendo-se uso de emulsificantes, hidrocolóides, ovos e outros aditivos para a melhora do produto final (Hosseini et al., 2018).

É comum que muitas pessoas que precisam consumir PSG já tenham consumido as versões com glúten, ou seja, pessoas que foram diagnosticadas com alguma condição que as impede de consumir glúten já na fase adulta, o que acaba gerando uma expectativa quanto às características sensoriais dos mesmos, tornando a aceitação desses produtos mais difícil (Dowd et al., 2014; Khoury et al., 2018).

O contrário também pode ser considerado verdadeiro, já que, conforme um estudo, ao mesmo tempo que a maior parte dos participantes estavam satisfeitos com os PSG, 73,6\% deles nunca haviam consumido glúten. Apenas 3,4\% estavam insatisfeitos com o sabor e $14 \%$ com a textura. Além disso, houve uma grande relação entre pessoas insatisfeitas com as características sensoriais e a dificuldade em encontrar PSG. Nesse mesmo estudo, o PSG que os participantes mais gostariam que fosse desenvolvido com melhor sabor foi o pão (do Nascimento et al., 2014). Fica perceptível, portanto, o avanço progressivo dos PSG, mas ainda insuficiente, sendo esse um grande fator para a não adesão intencional à dieta. 


\subsection{Fatores relacionados ao indivíduo}

Existem ainda fatores individuais que podem ser capazes de influenciar a adesão à dieta, tais como sexo, idade, educação e saúde mental (Wieser et al., 2021).

Quanto ao sexo dos indivíduos os estudos não são conclusivos. Uns não encontram diferenças, outros afirmam maior adesão do sexo feminino e outros do sexo masculino. Entretanto, quando a adesão foi maior nas mulheres, sugeriu-se que isso poderia ser decorrente dos sintomas mais leves nos homens, o que dificultaria a identificação da não adesão por meio dos sintomas, ou até mesmo por causa do controle mais rigoroso das mulheres sobre a dieta (Wieser et al., 2021).

Já com relação à idade, observou-se que, da infância à adolescência, quanto menor a idade maior a adesão. Para as crianças, essa adesão mais assertiva provavelmente é consequência de a manutenção da dieta ser realizada pelos pais; para os adolescentes, com menor adesão, a maior interação social e a consequente pressão social pode ser o fator que acaba interferindo nas escolhas alimentares (Wieser et al., 2021). Os adultos, portanto, têm maior probabilidade de seguir uma DSG (Lu et al., 2018).

Ademais, curiosamente um estudo realizado na China encontrou que participantes que possuíam ensino superior eram mais propensos a sentir dificuldade em seguir a dieta do que os de nível educacional inferior, o que os autores associaram à falta de alimentos sem glúten e à impossibilidade de preparar as próprias refeições em apartamentos das faculdades do país (Lu et al., 2018). Em contrapartida, outro estudo realizado nos Estados Unidos encontrou associação positiva entre maiores índices de adesão e maior escolaridade, que estava também associada ao maior conhecimento sobre a DSG, fator esse, como já visto anteriormente, fortemente relacionado à boa adesão (Villafuerte-Galvez et al., 2015).

Ressalta-se ainda, a importância de o indivíduo preparar as próprias refeições. Uma intervenção de educação nutricional baseada na culinária pode representar maiores índices de adesão e ainda melhoria na qualidade de vida incluindo a diminuição dos níveis de depressão e ansiedade, ainda que a pessoa tenha habilidades culinárias mínimas (Wolf et al., 2020); e indivíduos que cozinham as próprias refeições podem se sentir mais ajustados à DSG do que àqueles com outros métodos de manejo da dieta (Lu et al., 2018).

Por fim, há evidências de que existe uma associação significativa entre a baixa adesão e sintomas depressivos autorrelatados (Sainsbury et al., 2018), sintomas esses que já são comuns em pacientes com DC e, portanto, podem influenciar negativamente na adesão (Halmos et al., 2018; Muhammad et al., 2019). Desta maneira, um maior escore de qualidade de vida é importante para a adesão à dieta (Dimidi et al., 2021; Halmos et al., 2018), assim como estar associado a um grupo de suporte. Diante disso, é importante destacar a necessidade de acompanhamento não só de um nutricionista, mas também de um profissional da saúde mental, já que é imprescindível essa melhora na adesão e, consequentemente na saúde integral do indivíduo (Halmos et al., 2018).

Dessa forma, o acompanhamento profissional deve levar em consideração aspectos intrínsecos de cada indivíduo: qual o sexo? Participa de grupos de apoio? Possui qualidade de vida? Possui crenças limitantes sobre a DC ou sobre a DSG? Possui experiências com a adesão? Apenas assim, com um acompanhamento individualizado, é possível aumentar os níveis de adesão ultrapassando as barreiras próprias de cada pessoa (Dimidi et al., 2021).

\subsection{Fatores sociais}

Pessoas que apresentam distúrbios relacionados ao glúten não apresentam sinais externos deles, entretanto, as interações sociais, além de evidenciá-los, podem ser afetadas por causa das regras sociais. Além disso, revelações não planejadas sobre si mesmo têm capacidade de mudar permanentemente a forma como alguém é visto, por isso, muitos jovens optam pela não adesão consciente à dieta em prol da aceitação social (Olsson et al., 2009). 
Conforme se observa, seguir a dieta livre de glúten faz com que os jovens se sintam mais expostos e estranhos. Sentimentos como raiva, culpa e constrangimento são os mais expressados. Nessas situações, os jovens relatam ter que lidar com pessoas minimizando e negligenciando a sua condição por meio do incentivo ao consumo de glúten. Esses adolescentes relatam o desejo de que todos conhecessem suas necessidades dietéticas, de forma que agissem mais naturalmente sobre o assunto, além do desejo de que a disponibilidade de PSG fosse possível com o menor número de questionamentos. Nas escolas, esses jovens acreditam que uma pessoa treinada responsável por dietas especiais e refeições correspondentes à refeição normal seria ideal para diminuir o sentimento de que a DSG desvia das regras sociais (Olsson et al., 2009).

Além disso, uma revisão de escopo com foco em jovens e crianças com DC, apresentou uma série de atividades diárias relacionadas à alimentação que podem impactar na escolha alimentar desses indivíduos e prejudicar a adesão à dieta. Nessa idade, a independência parental, a pressão social e as novas responsabilidades associadas à própria alimentação somados à participação em situações cotidianas são dificuldades sociais capazes de influenciar o comportamento alimentar (Meyer \& Rosenblum, 2017).

Evidencia-se ainda a necessidade de se conhecer as atividades sociais nos diferentes estágios de desenvolvimento, tais como participar de festas de aniversário, comer em restaurantes, viajar, comer na casa de um amigo e fazer atividades na escola, para que, assim, os profissionais tenham condições de orientar da melhor forma possível seus pacientes a fim de que eles não sejam privados dessas atividades (Meyer \& Rosenblum, 2017).

Apesar de muitos estudos abordarem o impacto social sobre a adesão à dieta pelos jovens, um estudo realizado com adultos com DC diagnosticada na infância concluiu que $46 \%$ dos participantes restringem atividades do dia a dia por causa da dieta. Além disso, mesmo que os participantes tenham demonstrado uma adesão normalmente adequada, eles relataram dificuldade em manter consistência, especialmente ao viajar, comer em restaurantes ou visitar amigos (Leinonen et al., 2019).

Ademais, segundo uma pesquisa realizada na Noruega, das pessoas que consumiam glúten $30 \%$ o faziam não só por sentir desejo, mas também para se sentir normais e para socializar, sendo estes fatores até mais recorrentes para a não adesão do que o desconhecimento de que o produto tinha glúten (20\%) e o custo (12\%) (do Nascimento et al., 2014). Dessa forma, para manter a adesão, fica claro que as pessoas sentem a necessidade de fazer as refeições acompanhadas, especialmente quando a companhia é alguém que também não consome glúten, caso contrário, comer sozinho é que se torna um meio muito mais fácil de manter a adesão à dieta (Garnweidner-Holme et al., 2020). Prova disso, são os amigos que podem ser vistos como um fator que interfere negativamente na adesão, e isso pode acontecer justamente pela pouca informação e muitas vezes equivocada a respeito da dieta e da DC na sociedade como um todo (Antún et al., 2017).

\section{Considerações Finais}

Diante do exposto, ficam evidentes os inúmeros fatores capazes de interferir na adesão à DSG, tais como conhecimento a respeito da DSG, qualidade e custo dos produtos, questões de saúde mental e questões sociais.

Além disso, nem todas as pessoas que consomem glúten possuem os mesmos problemas para a adesão, de forma que o atendimento individualizado e detalhado deve ser o principal meio de monitoramento da adesão à dieta, para que se entenda os motivos e sejam feitas intervenções adequadas.

Dessa forma, os estudos posteriores devem focar justamente em intervenções (Antún et al., 2017; Villafuerte-Galvez et al., 2015), de forma a auxiliar na produção de estratégias e na compreensão da percepção dos pacientes sobre os problemas da adesão, construindo, assim, o caminho para melhores intervenções (Antún et al., 2017).

Assim, será possível potencializar o conhecimento em torno da DSG, e mais pessoas terão a oportunidade de tomar melhores decisões acerca da própria alimentação; além disso, será possível minimizar o impacto de situações sociais por meio de uma menor estigmatização sobre a dieta, e se poderá ainda alcançar a melhoria esperada em produtos e estabelecimentos. 
Research, Society and Development, v. 11, n. 2, e34411226008, 2022

(CC BY 4.0) | ISSN 2525-3409 | DOI: http://dx.doi.org/10.33448/rsd-v11i2.26008

\section{Referências}

Akobeng, A. K., \& Thomas, A. G. (2008). Systematic review: tolerable amount of gluten for people with coeliac disease. Alimentary Pharmacology \& Therapeutics. Artigo de K. A. Bascuñán (2017). Celiac disease: understanding the gluten-free diet. European journal of nutrition, 56(2), 449-459. https://doi.org/10.1111/J.1365-2036.2008.03669.X

Antún, M. C., González, V. B., de Ruggiero, M., Lava, M. Del P., Naranja, K., Alonso, N., \& Pedemonti, B. (2017). Factores que inciden en la adherencia a la dieta libre de gluten en adultos celíacos de la Ciudad Autónoma de Buenos Aires. Diaeta, 35(161), 10-18. http://www.scielo.org.ar/scielo.php?script=sci_arttext\&pid=S1852-73372017000400003\&lng=es\&nrm=iso\&tlng=es

Bengtsson, M. (2016). How to plan and perform a qualitative study using content analysis. NursingPlus Open, 2, 8-14. https://doi.org/10.1016/J.NPLS.2016.01.001

Borin Da Cunha, M., Kobal, D., \& Schneider, G. (2018). O Glúten em Questão. Química nova na escola. 40, 59-64. https://doi.org/10.21577/01048899.20160105

Case, S. (2005). The gluten-free diet: How to provide effective education and resources. Gastroenterology. Artigo de Z. Lu (2018). Gluten-free living in China: The characteristics, food choices and difficulties in following a gluten-free diet - An online survey. Appetite, 127, 242-248. https://doi.org/10.1053/J.GASTRO.2005.02.020

Codex Alimentarius Comission. (2008). Draft Revised Standard for Foods for Special Dietary Use for PersonsIntolerant to Gluten. http://www.jhnfa.org/CCNFSDU07.pdf

Cozzolino, S. M. F. (2016). Biodisponibilidade de nutrientes. Editora Manole.

Demirkesen, I., \& Ozkaya, B. (2020). Recent strategies for tackling the problems in gluten-free diet and products. Critical Reviews in Food Science and Nutrition. 1-27. Advance online publication. https://doi.org/10.1080/10408398.2020.1823814

Dimidi, E., Kabir, B., Singh, J., Ageridou, A., Foster, C., Ciclitira, P., Dubois, P., \& Whelan, K. (2021). Predictors of adherence to a gluten-free diet in celiac disease: Do knowledge, attitudes, experiences, symptoms, and quality of life play a role? Nutrition, 90, 111-249. https://doi.org/10.1016/J.NUT.2021.111249

Domínguez, J. (2017). Sobre las "bondades" de los alimentos sin gluten. Revista Cubana de Alimentación y Nutrición. 27(2), 483-487. https://www.medigraphic.com/cgi-bin/new/resumen.cgi?IDARTICULO=80177

do Nascimento, A. B., Fiates, G. M. R., dos Anjos, A., \& Teixeira, E. (2014). Gluten-free is not enough--perception and suggestions of celiac consumers International Journal of Food Sciences and Nutrition. Artigo de I. Demirkesen (2020). Recent strategies for tackling the problems in gluten-free diet and products. Critical Reviews in Food Science and Nutrition. 1-27. Advance online publication. https://doi.org/10.3109/09637486.2013.879286

Dowd, A. J., Tamminen, K. A., Jung, M. E., Case, S., Mcewan, D., \& Beauchamp, M. R. (2014). Motives for adherence to a gluten-free diet: a qualitative investigation involving adults with coeliac disease. Journal of Human Nutrition and Dietetics : The Official Journal of the British Dietetic Association. Artigo de H. Muhammad (2019). Identifying and improving adherence to the gluten-free diet in people with coeliac disease. Proceedings of the Nutrition Society, 78(3), 418-425. https://doi.org/10.1111/JHN.12203

Dunn, C., House, L., \& Shelnutt, K. P. (2014). Consumer Perceptions of Gluten-Free Products and the Healthfulness of Gluten-Free Diets. Undefined. Artigo de D. Khoury (2018). A Review on the Gluten-Free Diet: Technological and Nutritional Challenges. Nutrients, 10(10), 1410. https://doi.org/10.1016/J.JNEB.2014.04.280

Ellaway, A., \& Macintyre, S. (2000). Shopping for food in socially contrasting localities. British Food Journal. Artigo de H. Muhammad (2017). Adherence to a Gluten Free Diet Is Associated with Receiving Gluten Free Foods on Prescription and Understanding Food Labelling. Nutrients, 9(7), 705. https://doi.org/10.1108/00070700010310632/FULL/XML

Falcomer, A. L., Santos Araújo, L., Farage, P., Santos Monteiro, J., Yoshio Nakano, E., \& Puppin Zandonadi, R. (2018). Gluten contamination in food services and industry: A systematic review. Critical Reviews in Food Science and Nutrition. 60(3), 479-493. https://doi.org/10.1080/10408398.2018.1541864

Farage, P., de Medeiros Nóbrega, Y. K., Pratesi, R., Gandolfi, L., Assunção, P., \& Zandonadi, R. P. (2017). Gluten contamination in gluten-free bakery products: a risk for coeliac disease patients. Public Health Nutrition, 20(3), 413-416. https://doi.org/10.1017/S1368980016002433

Farage, P., Zandonadi, R. P., Gandolfi, L., Pratesi, R., Falcomer, A. L., Araújo, L. S., Nakano, E. Y., \& Ginani, V. C. (2019). Accidental Gluten Contamination in Traditional Lunch Meals from Food Services in Brasilia, Brazil. Nutrients, 11(8), 1924. https://doi.org/10.3390/NU11081924

Garnweidner-Holme, L., Sende, K., Hellmann, M., Henriksen, C., Lundin, K. E. A., Myhrstad, M. C. W., \& Telle-Hansen, V. H. (2020). Experiences of managing a gluten-free diet on multiple levels of society: a qualitative study. BMC Nutrition, 6(1), 65. https://doi.org/10.1186/S40795-020-00390-3

Gutowski, E. D., Weiten, D., Green, K. H., Rigaux, L. N., Bernstein, C. N., Graff, L. A., Walker, J. R., Duerksen, D. R., \& Silvester, J. A. (2020). Can individuals with celiac disease identify gluten-free foods correctly? Clinical Nutrition ESPEN, 36, 82-90. https://doi.org/10.1016/J.CLNESP.2020.01.012

Halmos, E. P., Deng, M., Knowles, S. R., Sainsbury, K., Mullan, B., \& Tye-Din, J. A. (2018). Food knowledge and psychological state predict adherence to a gluten-free diet in a survey of 5310 Australians and New Zealanders with coeliac disease. Alimentary Pharmacology \& Therapeutics, 48(1), 78-86. https://doi.org/10.1111/APT.14791

Hanci, O., \& Jeanes, Y. M. (2019). Are gluten-free food staples accessible to all patients with coeliac disease? Frontline Gastroenterology, 10(3), 222-228. https://doi.org/10.1136/FLGASTRO-2018-101088

Hosseini, S. M., Soltanizadeh, N., Mirmoghtadaee, P., Banavand, P., Mirmoghtadaie, L., \& Shojaee-Aliabadi, S. (2018). Gluten-free products in celiac disease: Nutritional and technological challenges and solutions. Journal of Research in Medical Sciences : The Official Journal of Isfahan University of Medical Sciences, 23(1), 109. https://doi.org/10.4103/JRMS.JRMS_666_18 
Research, Society and Development, v. 11, n. 2, e34411226008, 2022

(CC BY 4.0) | ISSN 2525-3409 | DOI: http://dx.doi.org/10.33448/rsd-v11i2.26008

Jamieson, J. A., \& Gougeon, L. (2019). Adults following a gluten-free diet report little dietary guidance in a pilot survey exploring relationships between dietary knowledge, management, and adherence in Nova Scotia, Canada. Nutrition Research, 66, 107-114. https://doi.org/10.1016/J.NUTRES.2019.02.005

Khoury, D. el, Balfour-Ducharme, S., \& Joye, I. J. (2018). A Review on the Gluten-Free Diet: Technological and Nutritional Challenges. Nutrients, 10(10), 1410. https://doi.org/10.3390/nu10101410

Kim, H. S., Patel, K. G., Orosz, E., Kothari, N., Demyen, M. F., Pyrsopoulos, N., \& Ahlawat, S. K. (2016). Time Trends in the Prevalence of Celiac Disease and Gluten-Free Diet in the US Population: Results From the National Health and Nutrition Examination Surveys 2009-2014. JAMA Internal Medicine, 176(11), 1716-1717. https://doi.org/10.1001/JAMAINTERNMED.2016.5254

Lei $n$. 10.674, de 16 de maio de 2003 (2003). Obriga a que os produtos alimentícios comercializados informem a presença de glúten, como medida preventiva e de controle da doença celíaca. Brasília, DF. https://doi.org/10.674

Leinonen, H., Kivelä, L., Lähdeaho, M.-L., Huhtala, H., Kaukinen, K., \& Kurppa, K. (2019). Daily Life Restrictions are Common and Associated with Health Concerns and Dietary Challenges in Adult Celiac Disease Patients Diagnosed in Childhood. Nutrients, 11(8), 1718. https://doi.org/10.3390/NU11081718

Løvik, A., Skodje, G., Bratlie, J., Brottveit, M., \& Lundin, K. E. A. (2017). Diet adherence and gluten exposure in coeliac disease and self-reported noncoeliac gluten sensitivity. Clinical Nutrition, 36(1), 275-280. https://doi.org/10.1016/J.CLNU.2015.11.017

Lu, Z., Zhang, H., Luoto, S., \& Ren, X. (2018). Gluten-free living in China: The characteristics, food choices and difficulties in following a gluten-free diet An online survey. Appetite, 127, 242-248. https://doi.org/10.1016/J.APPET.2018.05.007

MacCulloch, K., \& Rashid, M. (2014). Factors affecting adherence to a gluten-free diet in children with celiac disease. Paediatrics \& Child Health. Artigo de H. Leinonen (2019). Daily Life Restrictions are Common and Associated with Health Concerns and Dietary Challenges in Adult Celiac Disease Patients Diagnosed in Childhood. Nutrients, 11(8), 1718. https://doi.org/10.1093/PCH/19.6.305

Madden, A. M., Riordan, A. M., \& Knowles, L. (2016). Outcomes in coeliac disease: a qualitative exploration of patients' views on what they want to achieve when seeing a dietitian. Journal of Human Nutrition and Dietetics, 29(5), 607-616. https://doi.org/10.1111/JHN.12378

Meyer, S., \& Rosenblum, S. (2017). Activities, Participation and Quality of Life Concepts in Children and Adolescents with Celiac Disease: A Scoping Review. Nutrients, 9(9), 929. https://doi.org/10.3390/NU9090929

Muhammad, H., Reeves, S., Ishaq, S., Mayberry, J., \& Jeanes, Y. M. (2017). Adherence to a Gluten Free Diet Is Associated with Receiving Gluten Free Foods on Prescription and Understanding Food Labelling. Nutrients, 9(7), 705. https://doi.org/10.3390/NU9070705

Muhammad, H., Reeves, S., \& Jeanes, Y. M. (2019). Identifying and improving adherence to the gluten-free diet in people with coeliac disease. Proceedings of the Nutrition Society, 78(3), 418-425. https://doi.org/10.1017/S002966511800277X

Olsson, C., Lyon, P., Hörnell, A., Ivarsson, A., \& Sydner, Y. M. (2009). Food that makes you different: the stigma experienced by adolescents with celiac disease. Qualitative Health Research. Artigo de I. Demirkesen (2020). Recent strategies for tackling the problems in gluten-free diet and products. Critical Reviews in Food Science and Nutrition. 1-27. https://doi.org/10.1177/1049732309338722

Oyarzún, A., Nakash, T., Ayala, J., Lucero, Y., \& Araya, M. (2015). Following Gluten Free Diet: Less Available, Higher Cost and Poor Nutritional Profile of Gluten-Free School Snacks. International Journal of Celiac Disease. Artigo de K. A. Bascuñán (2017). Celiac disease: understanding the gluten-free diet. European journal of nutrition, 56(2), 449-459. https://doi.org/10.12691/IJCD-3-3-3

Paganizza, S., Zanotti, R., D’Odorico, A., Scapolo, P., \& Canova, C. (2019). Is adherence to a gluten-free diet by adult patients with celiac disease influenced by their knowledge of the gluten content of foods? Gastroenterology Nursing, 42(1), 55-64. https://doi.org/10.1097/SGA.0000000000000368

Rostami, K., Bold, J., Parr, A., \& Johnson, M. W. (2017). Gluten-Free Diet Indications, Safety, Quality, Labels, and Challenges. Nutrients, 9(8), 846. https://doi.org/10.3390/NU9080846

Sainsbury, K., \& Marques, M. M. (2018). The relationship between gluten free diet adherence and depressive symptoms in adults with coeliac disease: A systematic review with meta-analysis. Appetite, 120, 578-588. https://doi.org/10.1016/J.APPET.2017.10.017

Sant'Ana, W. P., \& Lemos, G. C. (2020). Metodologia científica: a pesquisa qualitativa nas visões de Lüdke e André. Revista eletrônica Científica Ensino Interdisciplinar, 4(12), 531-541. http://35.238.111.86:8080/jspui/handle/123456789/488

Schultz, M., Shin, S., \& Coppell, K. J. (2017). Awareness of coeliac disease among chefs and cooks depends on the level and place of training. Asia Pacific Journal of Clinical Nutrition, 26(4), 719-724. https://doi.org/10.6133/apjcn.072016.03

Silvester, J. A., Weiten, D., Graff, L. A., Walker, J. R., \& Duerksen, D. R. (2016). Is it gluten-free? Relationship between self-reported gluten-free diet adherence and knowledge of gluten content of foods. Nutrition (Burbank, Los Angeles County, Calif.), 32(7-8), 777-783. https://doi.org/10.1016/J.NUT.2016.01.021

Singh, J., \& Whelan, K. (2011). Limited availability and higher cost of gluten-free foods. Journal of Human Nutrition and Dietetics. Artigo de D. Khoury (2018). A Review on the Gluten-Free Diet: Technological and Nutritional Challenges. Nutrients, 10(10), 1410. https://doi.org/10.1111/J.1365277X.2011.01160.X

Terazono, E. (2017). Healthy appetites drive jump in sales of gluten-free foods | Financial Times. https://www.ft.com/content/4ec0f2f2-2c0a-11e7-9ec8$168383 \mathrm{da} 43 \mathrm{~b} 7$

Tovoli, F., Masi, C., Guidetti, E., Negrini, G., Paterini, P., \& Bolondi, L. (2015). Clinical and diagnostic aspects of gluten related disorders. World Journal of Clinical Cases: WJCC, 3(3), 275-284. Artigo de D. el Khoury (2018). A Review on the Gluten-Free Diet: Technological and Nutritional Challenges. Nutrients, 10(10), 1410. https://doi.org/10.12998/WJCC.V3.I3.275 
Research, Society and Development, v. 11, n. 2, e34411226008, 2022

(CC BY 4.0) | ISSN 2525-3409 | DOI: http://dx.doi.org/10.33448/rsd-v11i2.26008

Villafuerte-Galvez, J., Vanga, R. R., Dennis, M., Hansen, J., Leffler, D. A., Kelly, C. P., \& Mukherjee, R. (2015). Factors governing long-term adherence to a gluten-free diet in adult patients with coeliac disease. Alimentary Pharmacology \& Therapeutics. Artigo de H. Muhammad (2017). Adherence to a Gluten Free Diet Is Associated with Receiving Gluten Free Foods on Prescription and Understanding Food Labelling. Nutrients, 9(7), 705. https://doi.org/10.1111/APT.13319

Wieser H. (2007). Chemistry of gluten proteins. Food microbiology. Artigo de D. el Khoury (2018) A Review on the Gluten-Free Diet: Technological and Nutritional Challenges. Nutrients, 10(10), 1410. https://doi.org/10.1016/j.fm.2006.07.004

Wieser, H., Ruiz-Carnicer, Á., Segura, V., Comino, I., \& Sousa, C. (2021). Challenges of Monitoring the Gluten-Free Diet Adherence in the Management and Follow-Up of Patients with Celiac Disease. Nutrients, 13(7), 2274. https://doi.org/10.3390/NU13072274

Wolf, R. L., Morawetz, M., Lee, A. R., Koch, P. A., Contento, I. R., Zybert, P., Green, P. H. R., \& Lebwohl, B. (2020). A Cooking-Based Intervention Promotes Gluten-Free Diet Adherence and Quality of Life for Adults with Celiac Disease. Clinical Gastroenterology and Hepatology: The Official Clinical Practice Journal of the American Gastroenterological Association, 18(11), 2625-2627. https://doi.org/10.1016/J.CGH.2019.09.019 